\title{
XML-Based Management of Networks and Services
}

\author{
Aiko Pras, Jürgen Schönwälder, and Olivier Festor
}

Despite the fact that network operators and manufacturers face a major financial crisis, the size and importance of the Internet continue to grow at amazing speeds. At the core of the Internet, optical switches are replacing traditional switches, allowing data to flow at unprecedented speeds. At the edges, digital subscriber line (DSL) is becoming the technology of choice; within every home it is now possible to permanently connect multiple devices to the Internet. Wireless LAN (WLAN) hotspots are becoming commonplace, allowing people to connect to the Internet independent of whether they are flying in a plane, travelling on a train, or sitting on a terrace.

Considering the importance of the Internet in our economic and daily life, it is remarkable that technologies to manage the Internet get limited attention. Since the late 1980s, the Internet standard management framework has been based on the Simple Network Management Protocol (SNMP). In the '90s, the Internet Engineering Task Force (IETF) defined several new versions; the latest one, SNMPV3, has already been a full Internet standard for many years. However, despite its success, there is common agreement that SNMP technology will be unable to solve all future Internet management problems. Although it is likely that SNMP will remain the protocol of choice for many monitoring tasks, it is not the ideal technology for tasks such as configuration management. In fact, further progress in SNMP technology is unlikely to occur, and new versions of SNMP should no longer be expected.

As argued in the October 2003 issue of IEEE Communications Magazine, the evolutionary approaches to enhancing SNMP failed, so it seems the time has come for revolutionary approaches. Of such approaches, the most promising ones build on Extensible Markup Language (XML) and Web services technologies. In this issue of this magazine, an overview of research and recent developments in that area will therefore be presented.

The first article by George Pavlou et al. provides an overview of existing management technologies, such as open systems integration (OSI), SNMP, open distributed processing (ODP), and Common Object Request Broker Architecture (CORBA), and the potential of new technologies such as Web services. Based on the example of the TCP connection table, the authors show how to use these technologies for management purposes. Since there have been many discussions on the performance of XML and Web services technologies, the authors conclude with a performance comparison between SNMP, CORBA, and Web services.

Of key importance in network and service management is the ability to define management information in a standardized way. In the past several languages have been defined for that purpose; examples are Guidelines for the Definition of Managed Objects (GDMO), Structure of Management Information (SMI), and MOF. Because of the existence of many tools, XML seems to be the most appropriate syntactic framework to define such information in the future. The second article, by Jorge E. López de Vergara et al., discusses the use of XML for that purpose and proposes the use of ontology languages to enhance the semantic expressiveness of management definitions. In particular, they propose the use of OWL, the Web Ontology Language.

Even in revolutionary approaches, it remains important to allow graceful transition from 
current management information definitions toward future ones. The third article, by Torsten Klie and Frank Strau $\beta$, discusses such a transition and shows how existing SMI management information base (MIB) modules can be converted into new XML schema definitions. Furthermore, the article presents an SNMP-to-XML gateway that allows the retrieval of XML encoded management information in heterogeneous environments.

The fourth article, by Mi-Jung Choi et al., discusses the use of XML to facilitate IP configuration management. This topic is the subject of current standardization within the IETF NetConf Working Group. The article therefore starts with an overview of NetConf, and continues with presenting the specific XML-based configuration management system that was designed and implemented by the authors.

The fifth article, by Lawrence Menten, discusses experiences in exploring and designing XML-based network management solutions for products ranging from smallresource-limited devices to high-capacity server-hosted control plane monitoring systems. The article concludes that the adoption of XML representations and tools for device management will dramatically improve the completeness, flexibility, and robustness of the management infrastructure on devices with both large resources and small.

The last article, by Raouf Boutaba et al., is a good example of the potential use of Web services technology. The article presents the design and implementation of a user-controlled lightpath management system, which internally relies on XML and other Web services technologies, like SOAP and WSDL. This article provides an excellent proof that XML-based management is not only a topic for future research, but has already become an operational reality.

We would like to thank all authors who submitted articles to this feature topic. In total we received 35 articles, of which six were accepted. In particular, we would like to thank the more than 85 reviewers, without whose valuable comments this feature issue would not have been possible.

\section{Biographies}

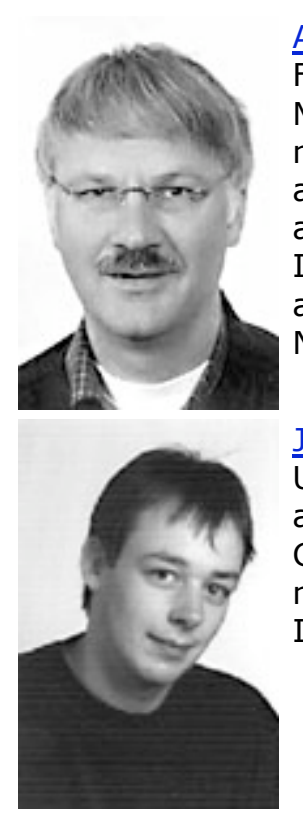

Aiko Pras [M] is associate professor at the University of Twente (UT), the Netherlands. From this university he received a Ph.D. degree in 1995 for his thesis, "Network Management Architectures." His current research interests include network management technologies, Web services, and network measurements and accounting. He has participated in many European and Dutch research projects, such as Mobile and Wireless, M2C, WASP, Candle, and Internet NG. He is a member of the Internet Research Task Force (IRTF) Network Management Research Group (NMRG) and technical co-chair of the Ninth IFIP/IEEE International Symposium on Integrated Network Management (IM 2005).

Jürgen Schönwälder $[\mathrm{M}]$ is a professor of computer science at the International University of Bremen, Germany. He received his diploma in computer science in 1990 and his doctoral degree in 1996 from the Technical University of Braunschweig, Germany. His research interests are network management, distributed systems, and network security. He is an active member of the IETF and chair of the NMRG of the IRTF.

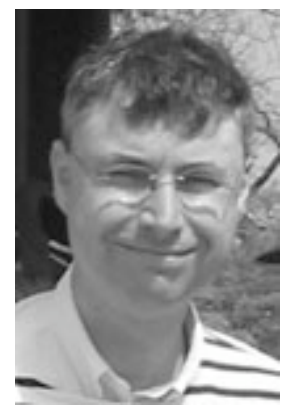

Olivier Festor $[\mathrm{M}]$ is a research director at INRIA, France, where he leads a research team on distributed network and service management. He has a Ph.D. degree (1994) and an Habilitation degree (2001) from Henri-Poincaré University, Nancy, France. His research interests are in the design of algorithms and models for automated and scalable management for highly dynamic environments such as ad hoc networks and peer-to-peer infrastructures. He is a member of the IRTF NMRG, and technical co-chair of IM 2005. 